\title{
Microstructure and mechanical properties of ultrafine-grained IF steel sheets produced by constrained groove pressing
}

\author{
Saeid Shahraki $^{a^{*}}$, Hadi Miyanaji ${ }^{\mathrm{b}}$ and Hossein Abdollahi ${ }^{\mathrm{a}}$
}

${ }^{a}$ Department of Mechanical Engineering, University of Zabol, Zabol, Iran

${ }^{b}$ Department of Industrial Engineering, University of Louisville, Louisville, USA

\begin{tabular}{l}
\hline A R T I C L EI N F O \\
\hline Article history: \\
Received 8 July, 2019 \\
Accepted 22 August 2019 \\
Available online \\
22 August 2019 \\
\hline Keywords: \\
SPD \\
CGP \\
Mechanical properties \\
Microstructure \\
\end{tabular}

\begin{abstract}
A B S T R A C T
In this paper, the severe plastic deformation method (SPD) known as constrained groove pressing (CGP) was applied to produce ultrafine-grained microstructure in IF steel sheets. In the CGP technique, samples are subjected to repetitive shear deformation under the plane strain deformation condition using the constrained grooved and flat dies. For assessment of microstructure and mechanical properties of the CGPed IF steel sheet, several testing such as: uniaxial tensile test, hardness measurements, scanning electron microscopy (SEM) and X-ray diffraction analysis have been utilized. Finally, it was observed that the grains are significantly refined after the 16-step pressing of the interstitial free (IF) steel sheets. The grains were reduced from $45 \mu \mathrm{m}$ to $243 \mathrm{~nm}$ after four passes.
\end{abstract}

(C) 2020 Growing Science Ltd. All rights reserved.

\section{Introduction}

In polycrystalline materials, the grain size plays a major role in establishing many crucial properties, including the strength and resistance to plastic flow. In general, materials with small grain sizes have several advantages over their coarse-grained counterparts, because they enjoy higher strength and other favorable properties including the potential for being used in superplastic forming operations at elevated temperatures. Such structures in polycrystalline materials are known as UltraFine-Grained(UFG), (Zhilyaev \& Langdon, 2008). Materials with UFG microstructures may be produced by either the "bottom-up" or "top-down" approaches. Severe Plastic Deformation (SPD) is a method for enhancement of mechanical properties and superplastic behavior with respect to the grain size reduction (Valiev et al., 2000). Several SPD methods have been developed such as: multidirectional forging (MDF), equal channel angular extrusion (ECAE), high pressure torsion (HPT), accumulative roll bonding (ARB), repetitive corrugation and straightening (RCS), constrained groove pressing (CGP), constrained groove rolling (CGR), etc. Two major SPD processes that are used for the production of ultrafine-grained microstructure in sheet metals are accumulative roll bonding (ARB) and constrained groove pressing (CGP). The ARB process, which has been invented by Saito et al. (1999), (Valiev et al., 1993), involves the repetitive bonding between two rolled plates, and if perfect bonding is not accomplished at the interface, the mechanical properties of the finished product may be diminished. Thus, ARB is considered less feasible for severe plastic deformation of sheet metals. Constrained groove pressing, originally proposed by Shin et al. (2002), has the advantage of imposing a more uniform severe plastic deformation on sheet metals, and it doesn't have the problem associated with bonding (Rafizadeh et al., 2009). Because of the mentioned advantages, this technique has attracted a great deal of attention from the researchers in the last decade for the fabrication of ultrafine-grained sheets. This technique has been widely applied on $\mathrm{Al}$ and $\mathrm{Cu}$ sheets, and its effectiveness in improving the mechanical properties and microstracture of these materials has been demonstrated by different researchers (Shin et al. 2002,

* Corresponding author. Tel.:+98-54-31232080-2

E-mail addresses: Saeid.Shahraki@uoz.ac.ir (S. Shahraki) 
Rafizadeh et al. 2009, Krishnaiah etal. 2009, Hosseini \& Kazeminezhad, 2009, Krishnaiah et al. 2005). However, there are very limited investigations about the effect of CGP on grain refinement of steel alloys (Khodabakhshi et al., 2010). In this study, for the first time, the CGP process was applied to interstitial free (IF) steel sheets. The interstitial free steel is an appropriate candidate for such an investigation, for several reasons. First, it is a representative single-phase BCC material. Second, due to a very-low carbon content, the absence of further interstitial solutes, and an appropriate texture, the IF steels exhibit an excellent deep drawbility, which is used in many automotive applications (Hadzima et al., 2007).

The CGP process proposed by Shin et al (2002), a grooved and a flat die are used to apply the deformation to the samples. In the grooved die, the width and height of the grooves are equal to the thickness of the specimen, and the angle of the grooves is 45 degrees. The schematic of the process has been shown in Fig 1. Since the groove pressing is carried out in such a way that the gap between the upper and lower portions of the dies is exactly the same as the thickness of the sample, the inclined sections of the sample in the grooved die are in pure shear strain, under the plane strain condition.

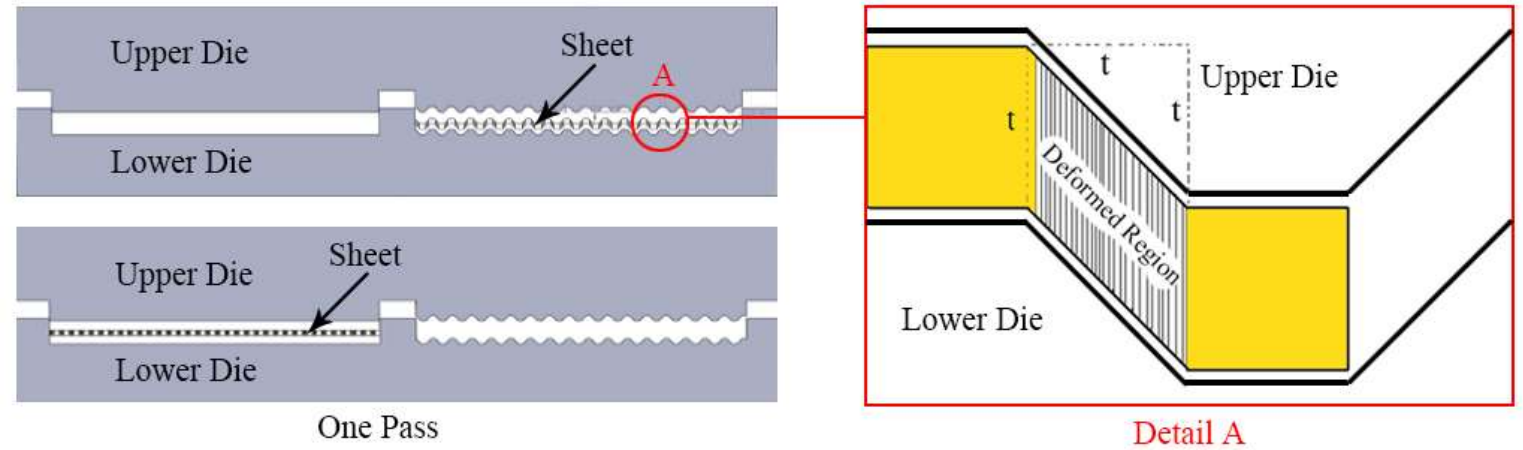

Fig. 1. Schematic of the dies and deformed region in the CGP process

In this paper the effects of the CGP process on the microstructure and mechanical properties of IF Steel have been evaluated. Firstly, the process has been accomplished on the sample. Then, after preparation of the samples, several experiments such as: tensile test, hardness measurements, SEM and X-ray diffraction analysis has been considered. Finally, the results of experiments were analyzed.

\section{Experimental procedure}

\subsection{Materials and method}

The designed die has been shown in Fig. 2. This die was prepared using the wire cut machining process, and it was constrained with a rectangular container during the pressing operations.

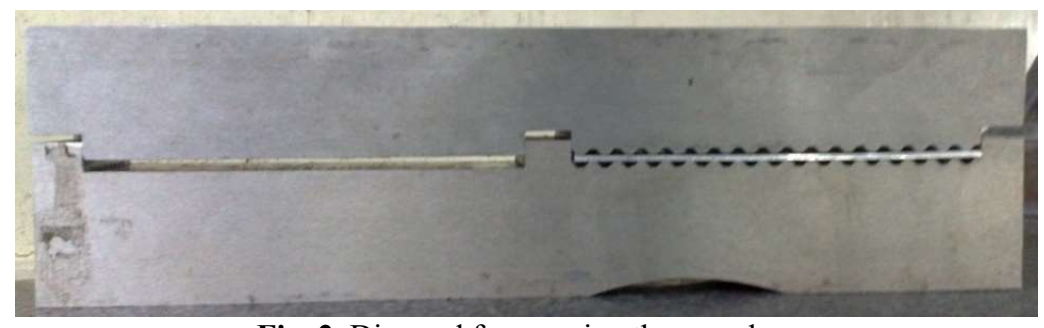

Fig. 2. Die used for pressing the samples.

The chemical composition of as-received IF Steel sheet is presented in Table 1. Firstly, IF steel specimens with the dimensions of $82 \times 40 \times 1.2 \mathrm{~mm}$ were prepared. Prior to the CGP process, the as-received sheets were annealed at a temperature of $750{ }^{\circ} \mathrm{C}$ for 30 minutes to obtain a recrystallized structure. Then, the prepared samples were pressed by applying the constrained groove pressing technique. The 16-step Pressing operations were conducted using a 15-ton hydraulic press machine operating at a constant pressing speed of $0.1 \mathrm{~mm} / \mathrm{s}$ at ambient temperature. The initial grain size of the annealed sheets was measured to be approximately $40 \mu \mathrm{m}$.

Table 1. Chemical composition of IF Steel sheet (\%).

\begin{tabular}{lllllll}
\hline $\mathbf{C}$ & $\mathbf{M n}$ & $\mathbf{S i}$ & $\mathbf{S}$ & $\mathbf{P}$ & $\mathbf{A l}$ & $\mathbf{P b}$ \\
$\mathbf{0 . 0 0 2}$ & 0.08 & 0.006 & 0.005 & 0.014 & 0.049 & 0.0003 \\
$\mathbf{T i}$ & $\mathbf{B}$ & $\mathbf{N b}$ & $\mathbf{C u}$ & $\mathbf{S n}$ & $\mathbf{C o}$ & $\mathbf{F e}$ \\
$\mathbf{0 . 0 4 4}$ & 0.0004 & 0.003 & 0.013 & 0.02 & 0.0009 & Base \\
\hline
\end{tabular}




\subsection{Mechanical properties}

To study the mechanical properties of the processed IF steel sheets, tensile and hardness tests were carried out on the specimens after each four pressings of the CGP process. The tensile test specimens were prepared such that the gage length was aligned with the longitudinal direction of the pressed sheets. Fig. 3 shows a prepared sample for the tensile test after the first pass. As it can be seen, the chosen dimensions for the tensile test specimens, according to the ASTM E8M standard, were $32 \times 6 \times 1.2 \mathrm{~mm}$. In order to examine the homogeneity of deformation, the Vickers hardness was measured along the central line of the transverse cross-section. A mean hardness value for each case was obtained from 10 measurements taken on each specimen.

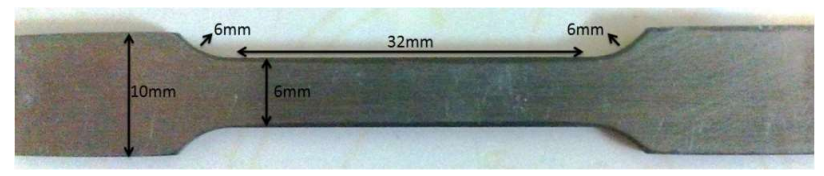

Fig. 3. The prepared sample for the uniaxial tensile test.

\subsection{Microstructure evaluation}

After the CGP process, the microstructure of the deformed sheets was investigated using both the Scanning Electron Microscopy and X-ray diffraction (XRD) techniques. For Scanning Electron Microscopy, the as-received and CGPed specimens were etched in a $2 \%$ nital solution for about 12 seconds, after being mounted and polished. To investigate the microstructure evaluation of the CGPed sample, The XRD measurements were obtained using a Philips X-ray diffractometer with $\mathrm{Cu} k \alpha$ radiation at $40 \mathrm{kV}$ and $30 \mathrm{~mA}$, which was set at the $2 \theta$ range from $10^{\circ}$ to $100 \circ$, and the results were analyzed using the Williamson-Hall method. Also, the X-ray patterns of the specimens were obtained at a step width of $0.02 \circ$. For further study on the microstructure of the CGPed sample, the XRD analysis was also used in addition to the SEM images to study the changes in the microstructure. Regarding the XRD patterns, it can be stated:

Two principal crystal imperfections in nanocrystalline materials (i.e., small crystallite size and lattice distortion) cause peak broadening compared with the Bragg peak, which results from perfect crystal diffraction. Accordingly, Grain size and lattice micro strain can be specified by measuring the deviation of line profile from perfect crystal diffraction. In addition, the environment in a commercial diffractometer is not ideal, and instrumental broadening arising from other effects must be extracted in advance (Zhang et al., 2003). In principle, the experimental profile is the convolution of the instrumental profile and the intrinsic profile (pure diffraction profile). The intrinsic profile can be obtained by unfolding the experimental profile. The simplified method is based on the assumptions that the intrinsic and instrumental profiles can be approximated by a bellshaped function, such as the Cauchy (Lorentz) or Gaussian function (Zhang et al., 2003). According to the assumption of Cauchy or Gaussian shape, the integral breadths can be described using the Gaussian-Gaussian (GG) relationship:

$$
B^{2}{ }_{\text {exp }} \cong B^{2}+B^{2}{ }_{\text {ins }},
$$

where $B_{\exp }, B$, and $B_{i n s}$ are the integral breadth of the experimental profile, intrinsic profile, and the instrumental profile, respectively. As it was described, grain size and lattice micro strain can be determined by measuring the deviation of line profile from perfect crystal diffraction. There are different reliable model-based approaches of X-ray diffraction line profile analysis, but in this study, the Williamson-Hall approach was used due to its simplicity and good reliable results. The Williamson-Hall equation can calculate the strain and crystalline size of materials as follows (Mukherjee et al., 2005):

$$
B \cos \theta=\frac{K \lambda}{d}+f(\varepsilon) \sin \theta
$$

where $B$ is the integral breadth of the XRD profile, $K$ is the Scherer constant, $d$ is the crystallite size, $\lambda$ is the wavelength of $\mathrm{X}$-ray, $\varepsilon$ is the lattice strain, $\theta$ is the Bragg angle, and $f$ is a defined function in Refs. ( Zhang et al., 2003; Mukherjee et al., 2005; Mukherjee et al., 2004). In the Williamson-Hall method, $B \cos \theta$ is plotted versus $\sin \theta$, and by applying the extrapolation approach, a line is fitted to this curve. Then, by estimating the slope of that line, the micro strain is obtained, and by finding the intersection of the line with the $B \cos \theta$ axis, the cell size is determined.

\section{Results and discussion}

\subsection{Evaluation of mechanical properties}

The results of tensile tests for the constrained groove pressed IF steel sheets at room temperature are shown in Fig. 4. The yield and ultimate strength of the CGPed specimens in the annealed condition were 155 Mpa 235 Mpa, respectively. According to the Figure, there is a sharp increase in the ultimate tensile strength (UTS) and yield strength (YS) of specimens after the first pressing pass. However, this increasing trend slows considerably in the second pass. The ultimate strength was increased to $382 \mathrm{MPa}$ and $391 \mathrm{Mpa}$ after the first and second pass, respectively. The increase of the YS and UTS of the CGPed specimens can be explained by work hardening during the initial pressing steps. As it was explained, the constrained groove pressing technique can impose large amounts of plastic deformation on the samples, which consequently leads to work hardening. 


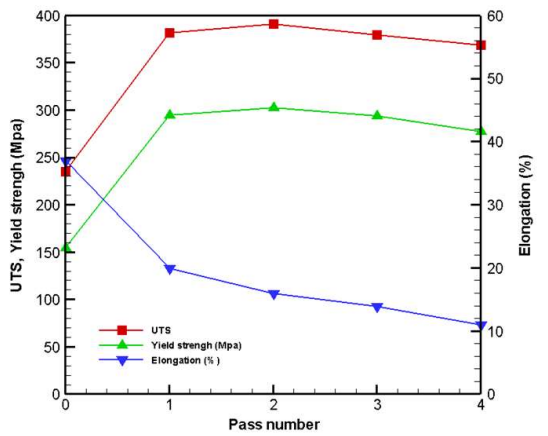

Fig. 4. Change of tensile properties of IF steel sheet with the number of passes

Subsequent pressing passes actually resulted in slightly lower tensile strengths, such that the UTS of the specimens decreased to $380 \mathrm{MPa}$ and $369 \mathrm{MPa}$ after the third and fourth pass, respectively. This drop in the tensile strength of the specimens may have two causes. The first cause is the saturation due to a high level of accumulated strain in the sheets. At this stage, the grains contain a large number of dislocations, and it is impossible to produce new dislocations in these grains. The second cause is the initiation of micro cracks on the surface of the specimens, especially at higher pass numbers, due to the friction and stress concentration in the corners of grooves (Shin et al., 2002; Krishnaiah et al., 2005, 2009; Tamimi et al., 2009). After the first pass, YS value increased to $295 \mathrm{Mpa}$. Like the UTS, there is a drop in the yield strength of the specimens after the second pass. As seen in Fig 4, elongation trend at fracture of the samples for all the passes was decreased. The elongation of the as-received sheets decreased from 0.35 to 0.10 after four passes. The decreasing elongation of the specimens can be justified by work hardening as well.

\subsection{Hardness Measurements}

The mean value of sheet hardness versus the number of pressing passes is shown in Fig. 5 (a). It can be observed that the hardness of the CGPed samples was increased by increasing the number of passes until the fourth pass. In the fourth pass, the samples hardness slightly were decreased. Also, for the examination of mechanical homogeneity, the hardness variation along the central line of the transverse cross-section of the samples has been shown in Fig. 5 (b) for each pass. It is clear that the imposed strain has become more uniform with the increasing number of passes. Since the hardness did not vary remarkably in the fourth pass, it implies that the imposed deformation by this technique is completely uniform throughout the sample. This means that the CGP process has the ability to produce ultrafine-grained sheets with good mechanical homogeneity.
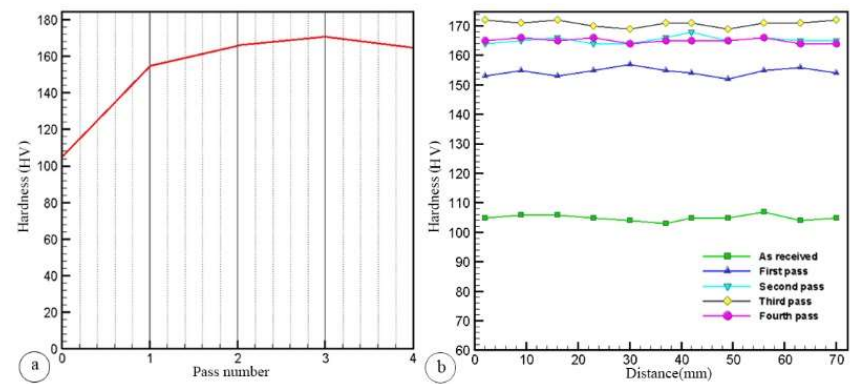

Fig. 5. Vickers hardness variation, a) Versus number of passes and b) Along the central line of the transverse cross-section.

\subsection{Microstructure evaluation}

The SEM microstructure of the annealed IF steel sheet is shown in Fig. 6 (a). It is clear from this figure that the grain size of the IF samples before the CGP process was about $45 \mu \mathrm{m}$. The grain shapes in these samples are spherical and coarse. It can be inferred from these figures that the grain size of the deformed samples decreases after the CGP passes.

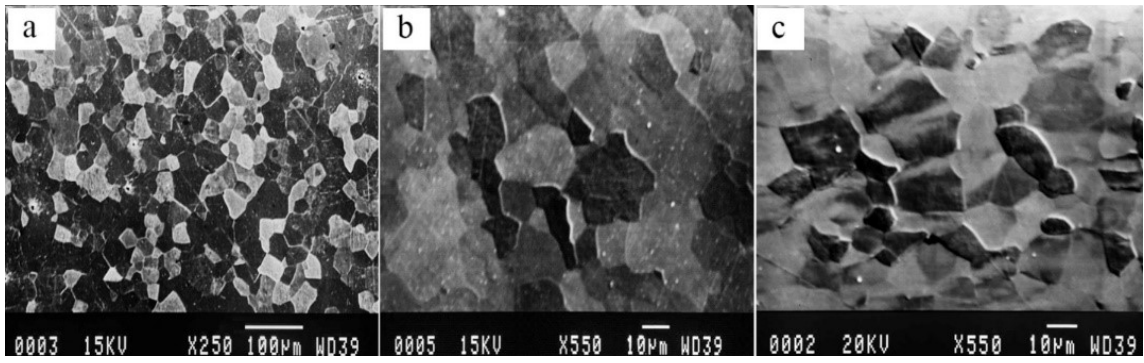

Fig. 6. The SEM microstructures of (a) Annealed sample, (b) After two passes, (c) After four passes. 
The X-ray diffraction (XRD) patterns obtained for the annealed sheet, one pass and four passes were shown in Fig. 7.
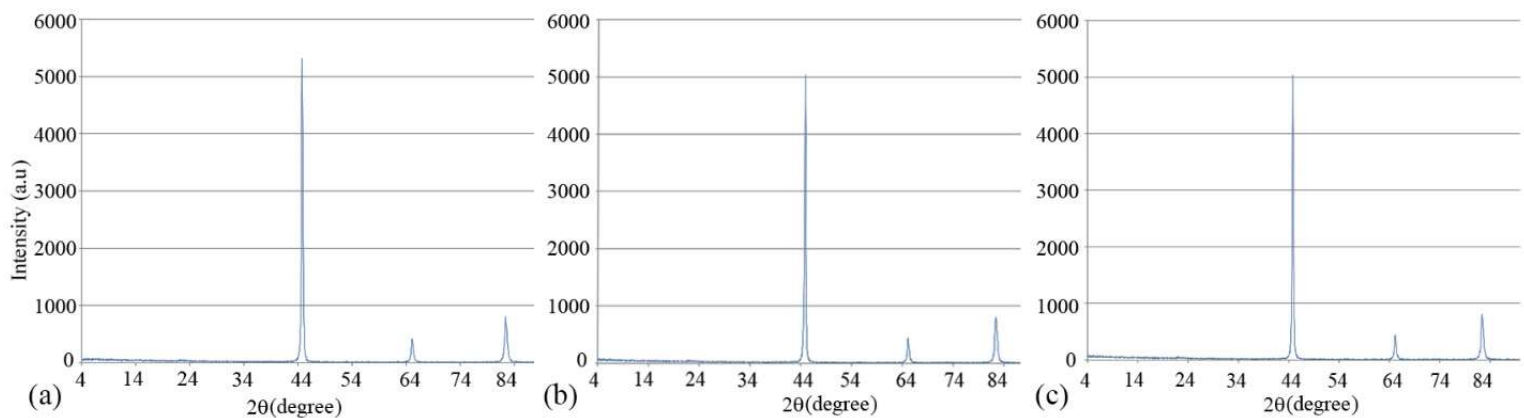

Fig. 7. X-ray diffraction (XRD) patterns obtained from, a) annealed sheet, b) CGP-processed sample after one pass and c) CGP-processed sample after four passes

In this research, the $B_{\text {ins }}$ of the instrument was measured to be 0.06 and then by using the Gaussuan-Gaussian relation and the known $B_{\exp }$ value, the amount of $B$ was calculated. It should be mentioned that in the present study, to examine the grain sizes of all the CGPed samples, the diffraction from three crystalline planes that have higher intensities compared to the other planes has been used. After performing the calculations in accordance with the mentioned explanations, the results are obtained according to Fig. 8 .

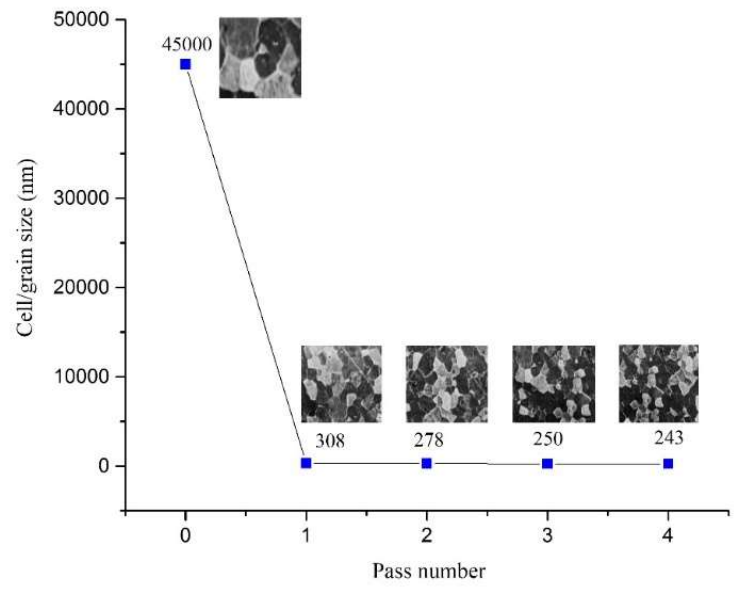

Fig. 8. The cell size versus CGP passes number.

As shown, in the first pass, the grains size were reduced from $45 \mu \mathrm{m}$ to $308 \mathrm{~nm}$. This reduction trend continues until the grain size reaches its final value of $243 \mathrm{~nm}$ in the fourth pass. To justify such a behavior, the theories of dislocation can be used. By considering the Orowan relation on a large time scale, the following relation is obtained between the imposed strain rate and the rate of dislocation generation (Roters et al., 2000):

$$
\rho^{+}=\gamma / b L
$$

Where $\rho^{+}$is the rate of dislocation density increasing, $L$ is the dislocation free path, $b$ is the Burgers vector and $\dot{\gamma}$ is the resolved strain rate. Since the cell size $(d)$ is proportionate to the dislocation density, $\rho$, in accordance with $d \propto \rho^{-0.5}$ (Hosseini et al. 2009, Estrin et al. 2008), considering the above relation, it seems that deformation can continually cause the reduction of the material structure's grain size; While, another type of dislocation reactions during plastic deformation (called dynamic recovery) results in a uniform rate of grain refinement. The main function of dynamic recovery is to eliminate dislocations, and since the rate of dynamic recovery is proportionate to the density of dislocations, the rate of dynamic recovery was increased with continued strain application and causes the reduction of the rate of grain refinement at higher strain values. Therefore, according to Fig 8, in light of the aforementioned explanations, it was totally acceptable to have a high speed of grain refinement during the initial pressing passes and a reduction of this speed during the subsequent passes.

\section{Conclusion}

In the present study, the IF steel sheets were subjected to severe plastic deformation (SPD) through a method called the constrained groove pressing (CGP), and a cumulative strain of 4.64 was imposed on the sheets during the four pressing passes. 
Then, the effect of the CGP process on the mechanical properties of the sheets was investigated. According to the research and obtained results, the following conclusion are drawn:

- The results showed that the tensile strength of the specimens increased until the second pass; however, Subsequent passes actually resulted in slightly lower tensile strengths, indicating some degrees of flow softening and the existence of surface microcarcks.

- The hardness of the specimens continuously increased with the increasing number of passes. Also, a loss of ductility was observed in all the processed sheets.

- The XRD analysis showed that the Grain size of the CGP-processed IF steel sheets decreased with the increasing number of passes. Eventually, with the application of this technique, a grain size of $243 \mathrm{~nm}$ was achieved in the IF steel sheets by imposing a strain magnitude of 4.64 .

\section{References}

Estrin, Y., Molotnikov, A., Davies, C. H. J., \& Lapovok, R. (2008). Strain gradient plasticity modelling of high-pressure torsion. Journal of the Mechanics and Physics of Solids, 56(4), 1186-1202

Hadzima, B., Janeček, M., Estrin, Y., \& Kim, H. S. (2007). Microstructure and corrosion properties of ultrafine-grained interstitial free steel. Materials Science and Engineering: A, 462(1-2), 243-247.

Hosseini, E., \& Kazeminezhad, M. (2009). Retracted: Nanostructure and mechanical properties of 0-7 strained aluminum by CGP: XRD, TEM and tensile test.

Hosseini, E., Kazeminezhad, M., Mani, A., \& Rafizadeh, E. (2009). On the evolution of flow stress during constrained groove pressing of pure copper sheet. Computational Materials Science, 45(4), 855-859

Khodabakhshi, F., Kazeminezhad, M., \& Kokabi, A. H. (2010). Constrained groove pressing of low carbon steel: Nanostructure and mechanical properties. Materials Science and Engineering: A, 527(16-17), 4043-4049.

Krishnaiah, A., Chakkingal, U., \& Venugopal, P. (2005). Applicability of the groove pressing technique for grain refinement in commercial purity copper. Materials Science and Engineering: A, 410, 337-340.

Krishnaiah, A., Chakkingal, U., \& Venugopal, P. (2005). Production of ultrafine grain sizes in aluminium sheets by severe plastic deformation using the technique of groove pressing. Scripta Materialia, 52(12), 1229-1233.

Mukherjee, P., Sarkar, A., Barat, P., Bandyopadhyay, S. K., Sen, P., Chattopadhyay, S. K., ... \& Mitra, M. K. (2004). Deformation characteristics of rolled zirconium alloys: a study by X-ray diffraction line profile analysis. Acta materialia, 52(19), 5687-5696.

Mukherjee, P., Sarkar, A., \& Barat, P. (2005). Microstructural changes in oxygen-irradiated zirconium-based alloy characterised by X-ray diffraction techniques. Materials characterization, 55(4-5), 412-417

Rafizadeh, E., Mani, A., \& Kazeminezhad, M. (2009). The effects of intermediate and post-annealing phenomena on the mechanical properties and microstructure of constrained groove pressed copper sheet. Materials Science and Engineering: A, 515(1-2), 162-168.

Roters, F., Raabe, D., \& Gottstein, G. (2000). Work hardening in heterogeneous alloys - a microstructural approach based on three internal state variables. Acta materialia, 48(17), 4181-4189.

Saito, Y., Utsunomiya, H., Tsuji, N., \& Sakai, T. (1999). Novel ultra-high straining process for bulk materials-development of the accumulative roll-bonding (ARB) process. Acta materialia, 47(2), 579-583.

Saito, Y., Utsunomiya, H., Tsuji, N., Sakai, T., \& Hong, R. G. (1999). Accumulative roll-bonding of 1100 aluminum. JOURNAL-JAPAN INSTITUTE OF METALS, 63, 790-795.

Shin, D. H., Park, J. J., Kim, Y. S., \& Park, K. T. (2002). Constrained groove pressing and its application to grain refinement of aluminum. materials Science and Engineering: A, 328(1-2), 98-103.

Tamimi, S., Ketabchi, M., \& Parvin, N. (2009). Microstructural evolution and mechanical properties of accumulative roll bonded interstitial free steel. Materials \& Design, 30(7), 2556-2562

Valiev, R. Z, Korznikov, A.V, Mulyukov, R.R. (1993) Mater. Sci. Eng., A168, pp. 141-148.

Valiev, R. Z., Islamgaliev, R. K., \& Alexandrov, I. V. (2000). Bulk nanostructured materials from severe plastic deformation. Progress in materials science, 45(2), 103-189.

Zhang, Z., Zhou, F., \& Lavernia, E. J. (2003). On the analysis of grain size in bulk nanocrystalline materials via X-ray diffraction. Metallurgical and Materials Transactions A, 34(6), 1349-1355.

Zhilyaev, A. P., \& Langdon, T. G. (2008). Using high-pressure torsion for metal processing: Fundamentals and applications. Progress in Materials science, 53(6), 893-979.

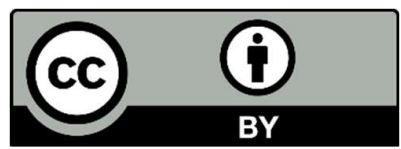

(C) 2020 by the authors; licensee Growing Science, Canada. This is an open access article distributed under the terms and conditions of the Creative Commons Attribution (CC-BY) license (http://creativecommons.org/licenses/by/4.0/). 